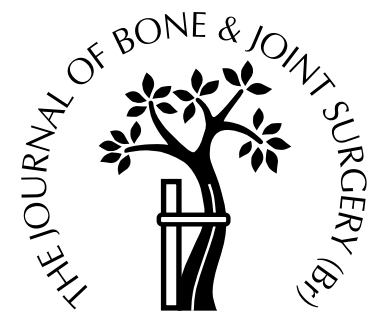

\title{
Toe-walking in children younger than six years with cerebral palsy
}

\author{
THE CONTRIBUTION OF SERIAL CORRECTIVE CASTS
}

J. Cottalorda, V. Gautheron, G. Metton, E. Charmet, Y. Chavrier

From the University of Medicine, Saint-Étienne, France

O

ur aim in this retrospective study was to analyse the value of serial corrective casts in the management of toe-walking in children aged less than six years with cerebral palsy. A total of 20 children (10 hemiplegic and 10 diplegic) had elongation of the triceps surae by serial casting at a mean age of four years and one month. The mean passive dorsiflexion of the foot with the knee in extension was $3^{\circ}(-10$ to $+5)$ and $12^{\circ}(0$ to +15$)$ with the knee in flexion. After removal of the cast passive dorsiflexion was $20^{\circ}(+10$ to +30$)$ with the knee in extension, and $28^{\circ}(+10$ to +35 ) with the knee in flexion. At a mean follow-up of 3.08 years $(2.08$ to 4.92$)$, passive dorsiflexion was $9^{\circ}$ $(-10$ to +20$)$ with the knee in extension and $18^{\circ}(0$ to $+30)$ with the knee in flexion.

Serial corrective casts are useful for the treatment of equinus in young children as the procedure is simple and the results are at least equal to those of other non-operative techniques. It is a safe alternative to surgical procedures especially in young children. If the equinus recurs operation can be undertaken on a tendon which is not scarred.

J Bone Joint Surg [Br] 2000;82-B:541-4.

Received 12 May 1999; Accepted after revision 27 October 1999

Equinus deformity of the ankle, defined as limitation of passive dorsiflexion beyond the neutral position, is common in children with cerebral palsy $(\mathrm{CP})$. ${ }^{1}$ It may be due to abnormal shortening of muscle, to a dynamic deformity caused by an exaggerated stretch reflex in the calf muscles or to a combination of both. ${ }^{2,3}$ In either case, the result is a

J. Cottalorda, MD, Attending Surgeon

Y. Chavrier, MD, Professor

Orthopaedic Paediatric Surgery Department

V. Gautheron, MD, Professor

G. Metton, MD, Consultant

E. Charmet, MD, Consultant

Physical Medecine and Rehabilitation Department

University of Medicine, Saint-Étienne, France.

Correspondence should be sent to Dr J. Cottalorda at Service de Chirurgie Infantile, Hôpital Nord, Avenue Albert Raimond, 42055 Saint-Étienne Cedex 2, France.

(C)2000 British Editorial Society of Bone and Joint Surgery

0301-620X/00/410188\$2.00

VOL. 82-B, No. 4, MAY 2000 characteristic toe-walking gait, which is clumsy and inefficient. ${ }^{4}$ Treatment is controversial, with some reports describing non-operative therapies to reduce contractures of the triceps surae, ${ }^{4-13}$ and others recommending surgical lengthening of tendo Achillis. ${ }^{1,3-16}$ The goal is to obtain and maintain a plantigrade foot, to prevent skeletal deformity and to improve gait. ${ }^{17}$ Non-operative treatments for equinus include physiotherapy, intramuscular injections of alcohol or botulinum toxin A, ankle-foot orthoses and serial casts. The aim is to postpone surgery on the calf until after the age of six years. ${ }^{6}$ Early surgery may have an unpredictable outcome, and recurrent equinus is related to the age at operation. $^{1,3,14,15}$ Hence, successful non-operative treatment to delay surgery may be worthwhile. ${ }^{6}$ Tardieu et al ${ }^{12}$ showed that prolonged maintenance of a muscle in the shortened position, either passively or by sustained contraction, as in spasticity, was a major cause of contracture. They also showed improvement in contracture of the triceps surae after serial casting for three weeks. Our aim was to analyse the place of serial casting in toe-walking children with $\mathrm{CP}$ who were aged less than six years.

\section{Patients and Methods}

Between April 1993 and February 1999, we carried out 65 elongations of the triceps surae using serial corrective casts in patients with CP. We analysed retrospectively the results in 30 feet of 20 patients ( 11 boys and 9 girls) aged less than six years with a minimum follow-up of 2.08 years. They were selected from the children with $\mathrm{CP}$ attending our department. Ten were hemiplegic and ten diplegic. The criteria for selection were: (1) spastic diplegia or hemiplegia; (2) the ability to be able to walk independently without aids for the upper limbs or a rollator walker; (3) a toe-walking gait; and (4) no static contracture of the triceps surae causing more than $10^{\circ}$ of fixed equinus with the knee extended and no static varus or valgus deformity of the ankle.

Their mean age was 4 years 1 month (2 years 4 months to 5 years 11 months). None had undergone previous orthopaedic surgery. The passive range of ankle movement was measured with a goniometer with the knee in extension and in flexion. The mean passive dorsiflexion before casting was $3^{\circ}(-10$ to +5$)$ with the knee in extension and $12^{\circ}$ $(0$ to +15$)$ with the knee in flexion. 


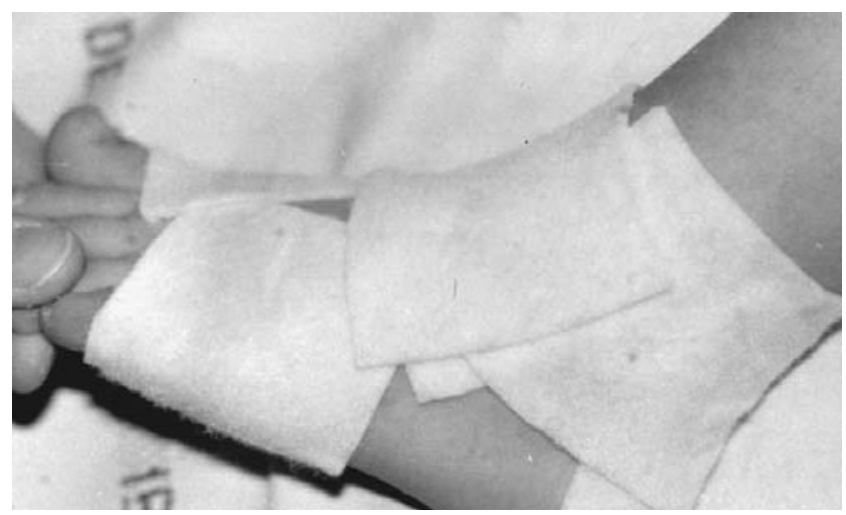

Fig. 1

Felt strips placed around the ankle.

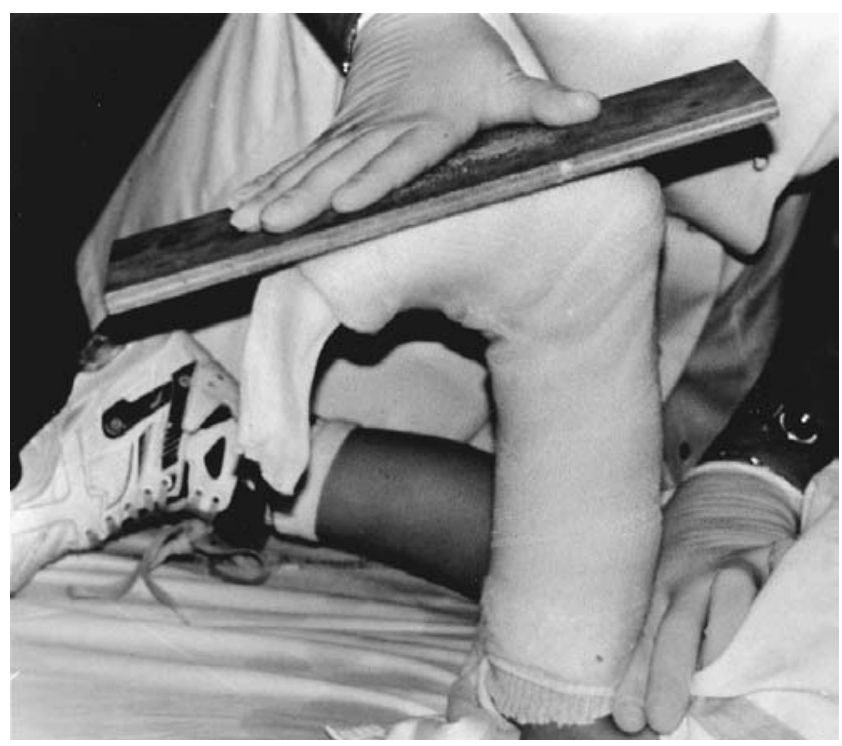

Fig. 3

Use of a foot board to prevent mid-foot dorsiflexion during stretching.

Technique of elongation by progressive casting. The resin casts were applied with the child relaxed in the prone position with the knee flexed. Preventive measures to avoid skin breakdown are essential. Several felt strips were placed all around the ankle (Fig. 1), which was then protected by stockinette and plaster wool (Fig. 2). The knee was held in flexion and the inverted foot in maximum dorsiflexion while an assistant applied the resin cast. Dorsiflexion was maintained with a footboard until the cast had dried in order to prevent mid-foot dorsiflexion (Fig. 3). The ankle was positioned in physiological valgus. All the elongations were carried out by the same senior author (JC). The extent of stretching achieved varied considerably from one child to another and was dependent on the patient's tolerance. The toes were placed in a neutral position by an extension of the cast beneath them. When a significant amount of dorsiflexion was obtained the cast was built up to compensate for this so that the child could walk. A rubber sole plate was applied and the children were sent

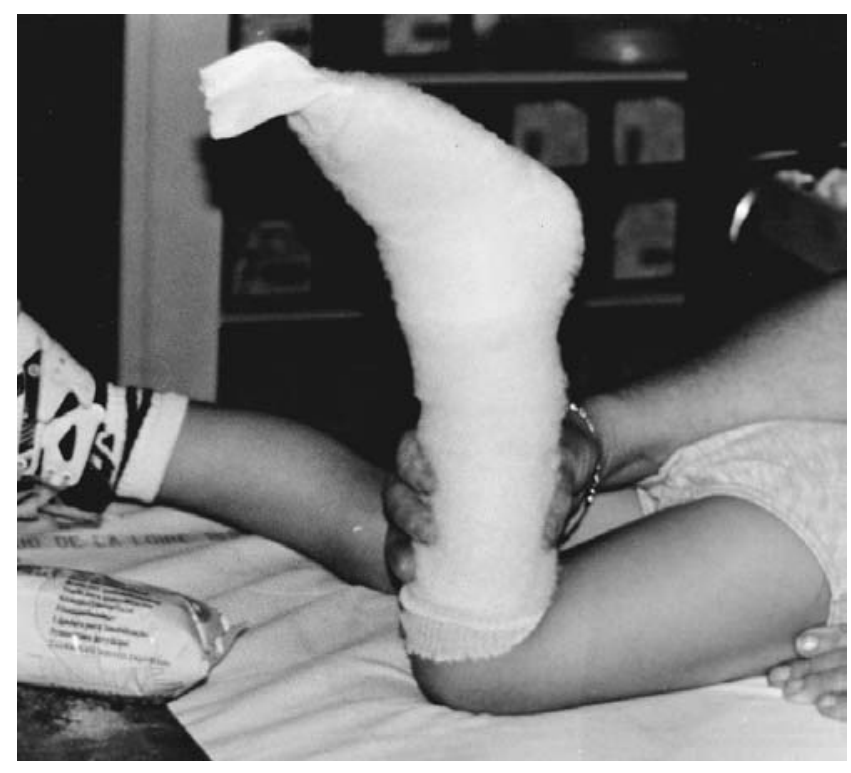

Fig. 2

View of a child in the prone position. Note the use of stockinette and plaster wool to prevent skin breakdown.

home non-weight-bearing for 24 hours. Since the knee was not immobilised, the children could walk in their casts. Three successive casts were applied over a period of three weeks. Immediately after removal of the cast a moulded plastic below-knee night splint, made before application of the cast with the foot in the neutral position, was used for a period of three months or more. Physiotherapy with repeated passive stretching of the tendon was continued for as long as possible.

The children were assessed clinically after treatment at one month, six months, one year and then every six months. Parental satisfaction was also assessed. The mean follow-up was for three years one month (2.08 to 4.92).

\section{Results}

There were three minor complications; one patient had a painful foot, one calf pain, and one inflammation of the skin, all of which resolved uneventfully. We measured the maximum angle of passive ankle dorsiflexion, obtained by serial corrective casts, with a goniometer. After removal of the cast passive dorsiflexion was $20^{\circ}(+10$ to +30$)$ with the knee in extension, and $28^{\circ}(+10$ to +35$)$ with the knee in flexion. We found no overcorrection. All children showed improved function with a flat-foot or heel/toe gait.

At the latest follow-up, the mean passive dorsiflexion was $9^{\circ}(-10$ to +20$)$ with the knee in extension and $18^{\circ}(0$ to +30$)$ with the knee in flexion. There were 15 feet with passive dorsiflexion beyond the neutral position and 15 below (i.e., equinus).

Feet with passive dorsiflexion below the neutral position. This occurred in 15 feet, nine of the 20 feet in the diplegic children and in six of the ten who were hemi- 
plegic. The range gained was lost over 12 months in three feet (two hemiplegic and one diplegic) which had received physiotherapy for three months or less, and over 18 months in 12 feet (four hemiplegic and eight diplegic).

In the early part of the study a second lengthening by serials casts was undertaken in nine feet 25 to 30 months after the first. Equinus deformity recurred in seven during the first year. The follow-up is too short to draw conclusions for the remaining two. Two have required aponeurotic lengthening of gastrocnemius, and the others may need surgery in the near future. In the remaining six feet treated later, no treatment of the recurrence has been undertaken because the functional problems were slight. We were disappointed by the failure of repeat serial casting in the previous nine and preferred to delay the decision to operate until the children were older.

Feet with passive dorsiflexion of the foot beyond the neutral position. Despite the fact that all 15 feet demonstrated passive dorsiflexion beyond neutral, seven had reverted to toe-walking at the last follow-up. Functional problems were, however, slight and a small improvement was still observed. Their parents preferred to delay any further treatment.

Parental satisfaction. The parents of 18 children thought their child had improved significantly, while those of the other two felt that the improvement was only moderate.

\section{Discussion}

A variety of methods to reduce equinus deformity because of muscle spasticity has been advocated. Injections of alcohol into the gastrocnemius muscles have been used for a long time, but the results are short-lived. ${ }^{2,4,5}$ Neurotomy or neurectomy of the gastrocnemius has been recommended for dynamic spastic equinus deformity ${ }^{18}$ without a triceps contracture. Recurrence of the deformity takes place between five and 17 months after this procedure. ${ }^{17}$ The effects of botulinum toxin A (BtA) last longer than injections of alcohol, possibly for three to six months. ${ }^{4,9}$ Koman et $\mathrm{al}^{9}$ suggested that the use of BtA may not completely avoid the necessity for surgical intervention but it may delay such treatment until the patient is older. The rate of recurrence after aponeurotic lengthening of the gastrocnemius is high (48\%), particularly if the initial operation is carried out before the age of five years. ${ }^{15}$ Recurrent equinus after lengthening of tendo Achillis is clearly related to the age of the child at operation in a number of series. ${ }^{1,3,14,15}$ Rattey et $\mathrm{al}^{1}$ reported a rate of recurrence of $50 \%$ in children aged less than three years at the time of operation. Successive casts are an alternative to these treatments.

Fulford $^{2}$ showed that a fixed deformity can be reversed and the length of the muscle restored by stretching with serial casts over a period of three or more weeks. Increased tone can decrease muscle length and conversely, stretching a relaxed muscle can increase the number of sarcomeres and muscle length. ${ }^{6}$
The hypothesis that pressure under certain plantar areas inhibits dynamic equinus ${ }^{6}$ has led to the use of 'tonereducing ankle-foot orthoses'. A small crossover study of two patients ${ }^{8}$ claimed an increased length of stride in tonereducing casts compared with standard casts. The effect was only noted while actually wearing the casts and not after treatment. ${ }^{6}$ Watt et $\mathrm{al}^{13}$ investigated the effect of a course of inhibitive casting and neurodevelopmental therapy for three-weeks on static muscle tonus, developmental skills, passive range of ankle dorsiflexion and pattern of gait. Two weeks after treatment, the passive range of ankle dorsiflexion and foot-floor contact in walking had improved significantly, but there was no significant change in static muscle tone or developmental skills. The improvement in ankle dorsiflexion and walking pattern lasted only five months. Sussman and Cusick ${ }^{10}$ and Sussman ${ }^{11}$ considered 'inhibitive casts' to have no direct therapeutic effect on muscle tone.

Corry et $\mathrm{al}^{6}$ compared BtA with stretching casts in the treatment of spastic equinus. Reduction of tone in the BtA group allowed a more prolonged improvement in passive dorsiflexion. Analysis of gait showed an improved mean ankle kinematic pattern in a subsection of both groups. This was maintained at 12 weeks in the BtA group, whereas the cast group relapsed.

Our results were better than those of Corry et $\mathrm{al}^{6}$ and Watt et al. ${ }^{13}$ In their studies, the feet were immobilised between three and six weeks in a neutral, plantigrade position with the toes dorsiflexed. In our technique, the serial corrective casts were applied in dorsiflexion over a period of three weeks. These differences in procedure may explain the results.

In our study, there was no significant difference in the recurrence of equinus deformity between diplegic (nine feet out of 20) and hemiplegic children (six feet out of 10). It was not possible to compare our results for recurrence with those in the literature since we were unable to find another study using serial casts which separated the children into different groups according to the aetiology of their cerebral palsy (quadraplegic, diplegic or hemiplegic).

It is difficult to maintain correction after removal of the cast since the muscle imbalance persists. We therefore used night splints to prevent recurrence. Tardieu et al ${ }^{19}$ showed that there was no progressive contracture when the soleus was stretched for at least six hours a day but that it occurred when the stretching time was as brief as two hours. A below-knee splint does not control the gastrocnemius and the commonly associated contracture of the hamstrings, but it is comfortable and well tolerated by the children. The purpose of the splints is for support, not correction and therefore the below-knee night splints were made with the ankle in the neutral position.

Theoretically, the threshold angle at which a slight resistance is met when the ankle is slowly dorsiflexed can be used to determine dorsiflexion. The difference between this angle and the angle at which a strong resistance is encoun- 
tered gives a measure of the muscle contracture, independent of tendon length. ${ }^{19}$ The principal aim of treatment is to increase the passive dorsiflexion, because it interferes with stable stance and gait. ${ }^{12}$ In our study, we decided to use the angle at which a strong resistance is encountered because it is a simple and reliable technique.

Our results may appear disappointing. At the latest follow-up, equinus deformity had recurred in 15 feet and in 22 feet the children had returned to toe-walking. We believe that it is very important to explain clearly to the parents that the goal of treatment is not to avoid surgery, but to postpone it until the children are older. Our study has shown that elongation by serial corrective casts is a rapid, attractive and dependable method for correcting dynamic equinus or mild fixed equinus deformity in young children for a period of 18 months or more. If surgery becomes necessary later, lengthening can be undertaken on an unscarred tendon. ${ }^{7}$ If the parents have been advised that a relative temporary improvement, rather than a normal gait, is the aim of treatment, most will be satisfied.

Early surgery is not advised in cerebral palsy as the delayed maturation of the central nervous system characteristic of this condition may produce variable and unpredictable results. ${ }^{3}$ We therefore prefer one, or occasionally two, periods in serial corrective casts rather than surgery in young children.

Intensive physiotherapy is indispensable after casting, otherwise recurrence will occur very rapidly. Casting by itself is not an isolated therapy, but when used appropriately, can be a useful adjunct to a neurodevelopmental therapy programme and facilitate improvement in the motor skills of children with cerebral palsy. ${ }^{11}$

No benefits in any form have been received or will be received from a commercial party related directly or indirectly to the subject of this article.

\section{References}

1. Rattey TE, Leahey L, Hyndman J, Brown DC, Gross M. Recurrence after Achilles tendon lengthening in cerebral palsy. J Pediatr Orthop 1993;13:184-7.
2. Fulford GE. Surgical management of ankle and foot deformities in cerebral palsy. Clin Orthop 1990;253:55-61.

3. Graham HK, Fixsen JA. Lengthening of the calcaneal tendon in spastic hemiplegia by the white slide technique: a long-term review. J Bone Joint Surg [Br] 1988;70-B:472-5.

4. Bleck EE. Cerebral palsy hip deformities: is there a consensus? II: botulinum toxin A: a clinical experiment. J Pediatr Orthop 1994;14: 281-2.

5. Carpenter EB, Seitz DG. Intramuscular alcohol as an aid in management of spastic cerebral palsy. Dev Med Child Neurol 1980;22: 497-501.

6. Corry IS, Cosgrove AP, Duffy CM, et al. Botulinum toxin A compared with stretching casts in the treatment of spastic equinus: a randomised prospective trial. J Pediatr Orthop 1998;18:304-11.

7. Cottalorda J, Gautheron V, Charmet E, Chavrier Y. Muscular lengthening of the triceps by successive casts in children with cerebral palsy. Rev Chir Orthop 1997;83:368-71.

8. Hinderer KA, Harris SR, Purdy AH, et al. Effects of tone reducing vs standard plaster-casts on gait improvement of children with cerebral palsy. Dev Med Child Neurol 1988;30:370-7.

9. Koman LA, Mooney JF, Smith BP, Goodman A, Mulvaney T. Management of spasticity in cerebral palsy with botulinum-A toxin: report of a preliminary, randomized, double-blind trial. $J$ Pediatr Orthop 1994;14:299-303.

10. Sussman MD, Cusick B. Preliminary report: the role of short-leg, tone-reducing casts as an adjunct to physical therapy of patients with cerebral palsy. Johns Hopkins Med J 1979;145:112-4.

11. Sussman MD. Casting as an adjunct to neurodevelopmental therapy for cerebral palsy. Dev Med Child Neurol 1983;25:804-5.

12. Tardieu G, Tardieu C, Colbeau-Justin P, Lespargot A. Muscle hypoextensibility in children with cerebral palsy: II. Therapeutic implications. Arch Phys Med Rehabil 1982;63:103-7.

13. Watt J, Sims D, Harckham F, et al. A prospective study of inhibitive casting as an adjunct to physiotherapy for cerebral palsied children. Dev Med Child Neurol 1986;28:480-8.

14. Lee CL, Bleek EE. Surgical correction of equinus deformity in cerebral palsy. Dev Med Child Neurol 1980;22:287-92.

15. Olney BW, Williams PF, Menelaus MB. Treatment of spastic equinus by aponeurosis lengthening. J Pediatr Orthop 1988;8:422-5.

16. O'Reilly DE, Carter EE. Surgical treatment of equinus in cerebral palsy. Dev Med and Child Neurol 1985;27:80-123.

17. Berard C, Sindou M, Berard J, Carrier H. Selective neurotomy of the tibial nerve in the spastic hemiplegic child: an explanation of the recurrence. J Pediat Orthop B 1998;7:66-70.

18. Doute DA, Sponseller PD, Tolo VT, Atkins E, Silberstein CE. Soleus neurectomy for dynamic ankle equinus in children with cerebral palsy. Am J Orthop 1997;26:613-6.

19. Tardieu C, Lespargot A, Tabary C, Bret MD. For how long must the soleus muscle be stretched each day to prevent contracture? Dev Med Child Neurol 1988;30:3-10. 